\title{
Facial expression recognition based on LBP and SVM decision tree
}

\author{
Mei-xia Yang ${ }^{1}$, Shu-mao Zheng $^{2}$, Yang Li ${ }^{1}$ \\ 1.The department of information engineering, Tianjin Modern Vocational Technology College, \\ Tianjin 300350 \\ 2.Tianjin Hwatsing Technology Company Limited, Tianjin 300350 \\ 497613494@qq.com
}

Keywords:facial expression recognition ; LBP ; SVM decision tree ; histogram

\begin{abstract}
In order to improve the recognition of facial expression recognition rate, facial expression recognition algorithm is based on a LBP and SVM decision tree. First will facial expression image is converted to LBP characteristic spectrum using LBP algorithm, and then the LBP characteristic spectrum into LBP histogram feature sequence, finally completes the classification and recognition of facial expression by SVM decision tree algorithm, and prove the effectiveness of the proposed method in the recognition of facial expression database in JAFFE.
\end{abstract}

\section{Introduction}

In recent years, with the development of artificial intelligence and human-computer interaction, the facial expression recognition and analysis of work more and more get people's attention, the purpose of facial expression recognition is the human face image information processing technology analysis of people's happiness, anger, sorrow and joy. In recent years, some classic facial expression recognition algorithms, mainly for facial expression recognition based on geometric feature extraction method is given priority to.using a projection points the way to extract the face the Euclidean distance between organs, to face the key geometrical relationship between organs, and combined with the prior knowledge of the people know to face structure characteristics of facial expression, such as: Brunelli in the literature[1] . In the literature [2], the use of a tracking KTL (kanade-Tucas -Tomasi) face expression way of feature point, to implement the face facial tracking under multiple expressions. The literature [3], Huang et . used a deformable template model combined with active contour model template for facial geometric facial features, then face facial expression characteristic is obtained. In ref. [4], the use of the combination of PCA and LDA method for facial expression recognition, obtained the good effect of facial expression recognition, and can effectively reduce the dimension of facial expression recognition problem. Facial expression recognition method based on geometric feature extraction to the advantage of more accurate description of facial expression characteristic, at the same time there is a problem, but this kind of method is expressed in characteristics when they lost some important identification and classification of information, resulting in expression recognition accuracy is not high. Based on the consideration of the above factors, this paper use the_Local Binary Pattern (LBP) and SVM decision tree (DT-SVM) a combination of face recognition research, it respectively as a facial expression feature extraction algorithms and implem entation facial expression recognition classifier.. In JAFFE facial expression for facial expression recognition experiment, with the results of the experiment proves the validity of this algorithm in facial expression recognition.

\section{LBP Feature Spectrum Histogram Sequence}

Local binary pattern (LBP) [4] is by Finland the scientist T.Ojala put forward a kind of effective operator, to describe the image texture features can be effective to the local texture feature of gray image feature extraction and measurement. The calculation process of the original LBP operator as follows: first of all to define a field window and defines the grey value of the center of the window as the threshold, the threshold respectively with eight points of the adjacent pixel values of 
sequence comparison, if the surrounding neighborhood point pixel gray value is greater than the threshold, will the position marked as 1 , otherwise marked as 0 . According to this method, you can gain an 8-bit binary encoding, and then give different weights in each position, finally will each binary encoding and the location of weights given by the multiplication, the product of the sum be an integer, the integer is the LBP values of the region. The calculation process as shown in the figure below:

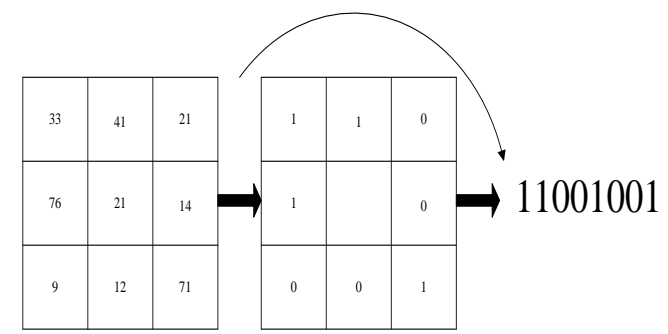

Figure 1 the calculation process of LBP

In order to overcome the LBP operator only within a radius of fixed neighborhood to calculate faults, T.O jala of LBP operator and others then to extend and improve, which USES the circular neighborhood instead of the original square neighborhood, its calculation method is: will a local texture distribution $\mathrm{T}$ assumed all pixel gray value within the local area of the joint distribution density, defined as:

$$
T=t\left(g_{C}, g_{0}, \ldots, g_{P-1}\right)
$$

Among them, $g_{C}$ on behalf of the gray levels of pixels in the neighborhood center, $g_{P}(p=0,1, \ldots, P)$ on behalf of the center pixel distribution evenly spaced around the pixel points, adjacent domain can be expressed as the coordinates of points:

$$
\left(x_{p}, y_{p}\right)=\left(x_{c}+R \cos (2 \pi p / P), y_{c}-R \sin (2 \pi p / P)\right)
$$

Among them, $\left(x_{c}, y_{c}\right)$ representative of the coordinates of the center pixel neighborhood, if the center pixel $g_{C}$ grayvalue from the field of removing, then you can put a local texture with center pixels and the pixels with neighboring pixels value difference between the joint probability distribution, the specific formula is as follows:

$$
T=t\left(g_{C}, g_{0}-g_{C}, \ldots, g_{P-1}-g_{C}\right)
$$

If the center pixel and the relationship between neighborhood pixels around the difference were independent of each other, will be (3) can be further stated as follows:

$$
T \approx t\left(g_{C}\right)\left(g_{0}-g_{C}, \ldots, g_{P-1}-g_{C}\right)
$$

In order to get the representation method of change is constant, relative to the scale can be (4) the following changes:

$$
T \approx t\left(s\left(g_{0}-g_{c}\right), \ldots, s\left(g_{p}-g_{c}\right)\right)
$$

Among them:

$$
s(x)= \begin{cases}1, & x>0 \\ 0, & x \leq 0\end{cases}
$$

The formula (6) for each of the $\boldsymbol{s ( \boldsymbol { g } _ { P } - \boldsymbol { g } _ { c } )}$ given different weights, commonly weight value is $2^{P}$, can get an image of the binary code. Calculation formula is shown below:

$$
\operatorname{LBP}\left(x_{c}, y_{c}\right)=\sum_{P=0}^{P-1} s\left(g_{p}-g_{c}\right) 2^{P}
$$

LBP operator is often used in [5] on facial expression

feature extraction, image below for the use of operator to extract the JAFFE facial expressions library LBP features rendering. 


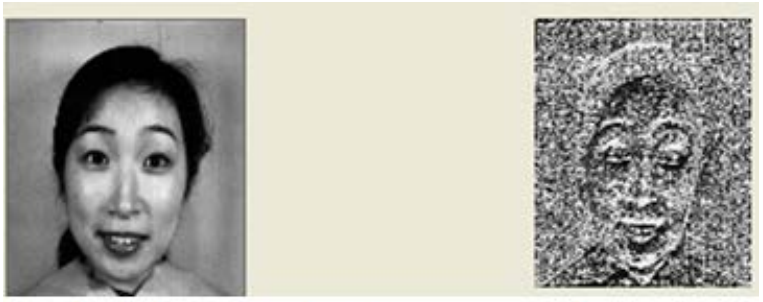

Figure 2 facial expression image LBP feature spectrum

LBP characteristic spectrum of facial expression only reflect the local features of a face, so it is not suitable for direct use of LBP characteristic spectrum to do facial expression recognition. If you want to get richer and more suitable characteristics, the general practice is to turn LBP characteristic spectrum into LBP histogram sequence[6], and then makes it as the discriminant features to do facial expression recognition. The specific extraction process of LBP extracted from the facial expression image histogram sequence as shown below.

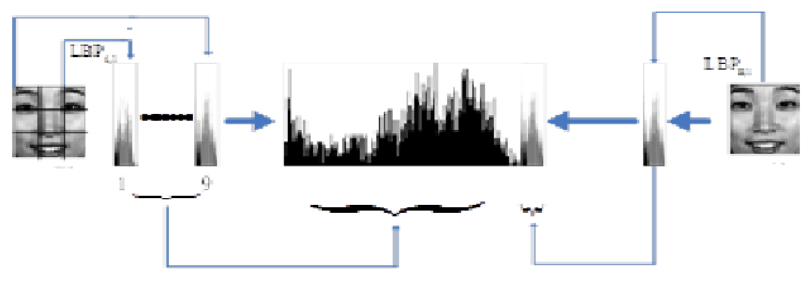

Figure 3 LBP face feature spectrum histogram sequence diagram of the extraction process

\section{SVM decision tree}

Support vector machine (SVM) [7-8], based on statistical, is proposed by V.Vapnik in 1995, which is a new generation machine learning algorithm. Because of its excellent solving the classification problem of nonlinear high dimensional space, it drew attention to many scholars. To solve the two samples of Separable problem in linear solution, suppose that there are some known data sets in $\mathrm{N}$ dimensional space such as formula (8) shows:

$$
\left\{x_{i,} y_{i} \mid x_{i}, \in R^{N}, y_{i} \in\{-1,1\}, i \in 1 \cdots m\right\}
$$

The goal is to find the maximum classification interval partitioning hyperplane, according to training samples. The optimal hyper plane equation can be expressed as

$$
f(x)=\operatorname{sgn} \sum_{i} a_{i} y_{i}\left(x_{i} \cdot x\right)+b
$$

The final SVM is transformed into a two programming form, as shown in the formula (10):

$$
\left\{\begin{array}{l}
\operatorname{Min}: \frac{1}{2} w^{T} w \\
S T: f(x)=\operatorname{sgn}\left\{a_{i} y_{i}\left(x_{i} \cdot x\right)+b\right\} \geq 1
\end{array}\right.
$$

The original SVM is only a two classifier, which can only solve the problem of two class classification of samples. However, the need to solve in the facial expression recognition and other pattern recognition problems are multi class classification problem. In order to solve the problem of multi classification algorithm SVM implementation, this paper adopts the method of SVM decision tree classification. This method will first divide all of the categories into two sub categories, and then further divide the two sub classes. so the cycle will continue until the subclass contains only one category. In this way, we will get a handstand two fork tree. Then, each decision node can be 
solved by using a SVM classifier. SVM decision tree only need to construct the k-1 SVM classifier for K classification problems.

\section{Face recognition experiments based on LBP and SVM decision tree}

The paper presents the facial expression recognition algorithm based on LBP and SVM decision tree. It is divided into two parts for training and recognition of facial expression.

The training part is mainly composed of three parts:

(1) Preconditioning: firstlyusing histogramequalization to correct facial expression avoid to be affected by the influence of light.

(2) Facial expression feature extraction: extracting LBP feature histogram sequence of facial expression, and taking it as the sequence of the distinguishing features of facial expression.

(3) Facial expression training: By using SVM method of decision tree to train LBP feature histogram sequence of facial expressions .

Facial expression recognition section also has three parts. The first two parts and the training part are the same, which all extract from face image preprocessing and face expression features. Only the last part of the testing is carried out on the facial expression recognition using SVM decision tree.

In order to verify facial expression recognition algorithm LBP and SVM based on decision tree in facial expression recognition and the validity proposed in this chapter for, the experiments of facial expression recognition in the JAFFE face database were selected. JAFFE facial expression database is composed of 10 Japanese women's210 face expression image, which everyone shows 7 different expressions (happy, angry, disgust, fear, sadness, surprise, calm). Each expression has 3 images. Below is a variety of facial expressions of a person in the JAFFE facial expression database.

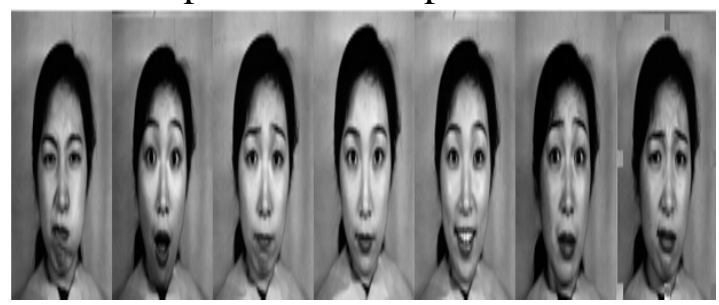

Figure 4 JAFFE facial expression database in a person's face expressions

In the experiment, to select 2 images of each person's each expression, a total of 140 pieces, the training set is composed of the facial expression. Training in the training sequence using LBP histograms of the training set of 140 face expression image SVM decision tree algorithm based on genetic algorithm, the final training a 7 root nodes of decision tree SVM, 7root node which is the 7 kind of facial expression.

In the recognition phase by using the method of KNN and SVM is based on a combination of tests on a variety of facial expression recognition, recognition of each expression achieved rate and compared to other algorithms as shown in table 1 :

Table 1 in this paper, the algorithm and other algorithms obtain recognition and speech recognition

\begin{tabular}{|c|c|c|c|c|c|c|c|c|}
\hline \multirow{2}{*}{ algorithm } & \multirow[b]{2}{*}{ calm } & \multirow[b]{2}{*}{ Happy } & \multicolumn{5}{|c|}{ Facial expression recognition rate $(\%)$} & \multirow[b]{2}{*}{ average, } \\
\hline & & & angry & surprised & sad & hate & fear & \\
\hline LBP & 87 & 85 & 80 & 76 & 69 & 66 & 65 & 75 \\
\hline LBP+DT-SVM & 93 & 92 & 92 & 90 & 80 & 75 & 73 & 88 \\
\hline This paper & 97 & 95 & 95 & 93 & 90 & 88 & 86 & 92 \\
\hline
\end{tabular}




\section{Conclusion}

The paper proposes a LBP and SVM decision tree based on the combination of facial expression recognition algorithm. The specific realization of facial expression recognition process is: first, to take the LBP histogram sequence of facial expression image as the distinguishing features of facial expression, and then, to adopt the SVM decision tree classification algorithm for classification and recognition of facial expression. In the experiment of facial expression recognition JAFFE facial expression database revealed 7 face recognition algorithm on the face, which can achieve an average recognition rate of $92.45 \%$.

\section{References}

[1] Brunelli R, Poggio T, Feature recognition; features versus templates[J]. IEEE Transactions on Pattern Analysis and Machine Intelligence, 1993, 15(10):1042-1052.

[2] Duan Hong, yi-min cheng, yi-xiao wang etc. Kanade - Lucas - Tomasi algorithm based face feature points tracking method [J]. Journal of computer-aided design and graphics, 2004, 16 (3) : 279-283.

[3] Huang C L, Chen C W. Human facial feature extraction for face interpretation[J]. Pattern Recognition,1992.25(12):1435-1444.

[4] hong-bo deng, jin lianwen. Based on local Gabor filter bank and PCA + LDA facial expression recognition algorithm [J]. Journal of image and graphics Newspaper, 2007, 12 (2) : 322-329.

[5] cheng, liu shi. For facial expression recognition of AVR and enhanced LBP feature selection methods [J]. Computer engineering and should be 1999, 45 (19) : 184-188.

[6] T.Ahonen, A.Hadid, M.Pietikainen. Face recognition with local binary patterns[C]. InProc of 8th European Conference on Computer Vision, Pargue, Czech,2004:469-481.

[7] Yang Changcheng TaoLiang. Performance comparison of several kinds of machine learning methods in face recognition, [J]. Computer engineering and application, 2009, (4) : 169-172.

[8] Takuya Inoue, Shigeo Abe. Fuzzy Support Vector Machines for Pattern Classification. Neural Networks,2001.Proceedings.IJCNN'01.International Joint Conference on, 1: 1449-1454. 